\title{
Qualitätsreport 2011: \\ Bericht über die Behandlungsqualität minimalinvasiver Methoden - Teil 2 Interventionelle Therapie (EVAR) von Aortenaneurysmen
}

\section{Quality Report 2011 of the German Society of Interventional Radiology (DeGIR) - Part 2 Endovascular Treatment of Aortic Aneurysms (EVAR)}

Autoren

Institute
L. J. Heuser ${ }^{1}$, C. N. Arnold ${ }^{2}$, D. Morhard ${ }^{3}$, M. Köhler ${ }^{4}$, W. Gross-Fengels ${ }^{5}$, A. Bücker ${ }^{6}$

Die Institutsangaben sind am Ende des Beitrags gelistet.

Key words
aorta
aneurysm
stents
arteriosclerosis
dissection
grafts
Quality management
EVAR
aortic aneurysm

eingereicht 13.5.2013

akzeptiert 22.5.2013

Bibliografie

Dol http://dx.doi.org/

10.1055/s-0033-1335736

Online-Publikation: 27.5.2013

Fortschr Röntgenstr 2013; 185:

709-719 @ Georg Thieme

Verlag KG Stuttgart . New York .

ISSN 1438-9029

Korrespondenzadresse

Prof. Dr. Lothar J. Heuser

Institut für Diagnostische und Interventionelle Radologie,

Neuroradiologie und

Nuklearmedizin,

Universitätsklinikum

Knappschaftskrankenhaus

$\mathrm{GmbH}$

In der Schornau 23-25

44892 Bochum

Tel.: ++49/234/2 993800

Fax: ++49/234/2993809

Lothar.Heuser@rub.de

\section{Zusammenfassung}

$\nabla$

Ziel: Analyse der Behandlungsqualität der endovaskulären Versorgung von Aortenaneurysmen anhand der Eingaben in das DeGIR-Qualitätssicherungssystem.

Material und Methoden: Die Daten des Jahres 2011 zur Behandlungsqualität der endovaskulären Versorgung von Aortenaneurysmen des freiwilligen DeGIR-Qualitätssicherungssystems wurden retrospektiv ausgewertet. Die Indikationsstellung, der Therapieprozess und die Ergebnisqualität wurden überprüft. Hinsichtlich der Ergebnisparameter wurden insbesondere der Therapieerfolg, die Komplikationsraten und die Strahlenexposition untersucht.

Ergebnisse: Von 82881 Datensätzen des DeGIRQualitätssicherungssystems des Jahres 2011 beschreiben 1167 Datensätze die Behandlung eines Aortenaneurysmas. Bei 12,4\% der Fälle handelte es sich um eine Notfallindikation. Die häufigste Indikation zur EVAR-Behandlung stellte mit 85\% das abdominelle Aneurysma dar. Der mediane Aortendurchmesser betrug 56,5 mm, 253 Fälle hatten eine Aortendurchmesser zwischen 50 und $55 \mathrm{~mm}$. Der technische Erfolg der EVAR-Behandlung lag bei $94,6 \%$. Die Komplikationsrate aller EVAR einschließlich der Notfallindikationen lag bei $4 \%$ mit einer Major-Komplikationsrate von $2,5 \%$. Elektiv behandelte Patienten wiesen eine Letalität von $0,34 \%$ auf. Bei bereits präinterventionell rupturierten Aneurysmen lag die Letalität bei $12 \%$. Die Mediane des Flächendosisprodukts und der Durchleuchtungszeit lagen bei $10676,5 \mathrm{cGy} \times \mathrm{cm}^{2}$ und 17,32 $\mathrm{min}$.

Schlussfolgerung: Die Datenauswertung des DeGIR-Qualitätssicherungssystems belegt eine sehr hohe technische Erfolgsrate der erfassten endovaskulären Behandlungen von Aortenaneurysmen bei gleichzeitig geringer Komplikationsrate. Um die Datenqualität noch weiter zu verbessern,

\section{Abstract \\ $\nabla$}

Purpose: To analyze the quality of treatment for endovascular aortic aneurysm repair using the data of the DeGIR quality management system.

Materials and Methods: A retrospective analysis of all data registered in the DeGIR quality management system of the year 2011 was performed regarding the treatment quality for endovascular aortic aneurysm repair. Registry of data within this system was voluntary. Quality aims of correct indication, treatment strategy and results were examined. Special interest was directed towards treatment success, complication rates and radiation exposure.

Results: Out of 82881 data sets from the year 2011 overall 1167 cases of EVAR were registered. $12.4 \%$ of these cases encompassed emergency treatments. The most frequent indication was an abdominal aneurysm with $85 \%$ of cases. The median aortic diameter was $56.5 \mathrm{~mm}$. 253 cases showed an aortic diameter between 50 and $55 \mathrm{~mm}$. Technical success was achieved in 94.6\% of all cases including emergency indications for aortic rupture. The overall complication rate of all cases was $4 \%$ with $2.5 \%$ major complications. Examining only the elective cases a mortality rate of $0.34 \%$ was found. EVAR of ruptured aneurysms yielded a mortality rate of $12 \%$. Median dose area product and fluoroscopy time were $10676.5 \mathrm{cGy} \times \mathrm{cm}^{2}$ und $17.32 \mathrm{~min}$ respectively.

Conclusion: Data analysis of the DeGIR quality management system proved a very high technical success rate for the registered cases of endovascular aortic aneurysm repair accompanied by a low complication rate. Improvement of data quality will need further mandatory fields within the software to be implemented.

Key Points: The voluntary DeGIR quality management system has reached a high acceptance among radiologists. Endovascular aortic aneurysm repair 
ist eine Modifikation der Datenerhebung mit Einführung weiterer Pflichtfelder bei der Eingabe notwendig.

Kernaussagen: Das freiwillige DeGIR-Qualitätssicherungssystem hat unter interventionell tätigen Ärzten eine hohe Akzeptanz gefunden. Die endovaskuläre Aneurysmabehandlung durch Radiologen besitzt eine hohe technische Erfolgsrate bei gleichzeitig sehr niedriger Komplikationsrate. by radiologists has shown a very high technical success rate and a very low complication rate.

\section{Einleitung}

$\nabla$

Die Deutsche Gesellschaft für Interventionelle Radiologie (DeGIR) erfasst in ihrem Qualitätssicherungssystem (DeGIR-QS-System) seit 1994 regelmäßig freiwillig eingegebene pseudonymisierte Behandlungsdaten von minimalinvasiven, radiologischen Interventionen [1]. Ziele sind die Messung, die Sicherung und die Verbesserung der Behandlungsqualität bei allen Verfahren der Interventionellen Radiologie [2, 3]. Die hierzu eingesetzte Software wurde kontinuierlich durch die Rückkopplung der Nutzer mehrfach in ihrer Benutzerfreundlichkeit verbessert, ergänzt und der Entwicklung einzelner interventioneller Therapiemethoden angepasst. So wurde im Jahr 2010 der Bereich „Aneurysmabehandlung (EVAR)“ komplett überarbeitet und ist in dieser aktuellen Version seit dem 1.1.2011 im Einsatz. Der nachfolgende Bericht gibt die erzielte Behandlungsqualität gemessen an den aufgestellten Qualitätszielen wieder.

\section{Struktur der Software und teilnehmende Institute $\nabla$}

Das zentrale Datenmanagementsystem wurde bereits in Teil 1 dieses Qualitätsreports [1] ausführlich beschrieben. An dieser Stelle sei deshalb nur darauf hingewiesen, dass die browsergestützte Dateneingabe jederzeit ( $24 \mathrm{~h} / 7 \mathrm{~d}$ ) erfolgen kann und die Zahl der zu registrierenden Datensätze nicht begrenzt ist. Eingabeschluss für das jeweilige Kalenderjahr ist der 28. Februar des Folgejahres. In jährlichen Abständen erfolgen Basis- und Sonderauswertungen sowie Änderungen, Ergänzungen und Korrekturen der einzelnen Software-Module.

Die Teilnahme am DeGIR-QS-System ist derzeit freiwillig. Für die zertifizierte Qualifikation in der Interventionellen Radiologie und für die Zertifizierung der radiologischen Kliniken und Institute als Partner von Gefäßzentren sind die Teilnahme und die Meldung von Mindestzahlen bei den entsprechenden Interventionen eine zwingende Voraussetzung. Dies ermöglicht der DeGIR, Zertifikate über die Qualifikation von Einzelpersonen und von Ausbildungsstätten ausschließlich an entsprechend qualifizierte und erfahrene Personen und Kliniken auszugeben.

\section{Auswertung der Daten \\ $\nabla$}

Für die Datenanalyse ist es wichtig, dass die jeweiligen Qualitätsziele definiert werden. Für die Behandlung von Aortenaneurysmen wurden folgende Qualitätsziele festgelegt:

\section{Indikation:}

- elektiver Eingriff/Notfall

- interdisziplinäre Indikationsstellung

- Aneurysmalokalisation (thorakal, thorakoabdominell, abdominell)

- Aneurysma-Art (verum, spurium, dissecans)
- kompliziertes Aneurysma (gedeckte/freie Perforation, Inflammation/Infektion)

- Durchmesser

\section{Prozess:}

- Zugang (operativ, perkutan)

- Prothesentyp (Rohr, aorto-mono-/biiliakal, fenestriert, Seitenast)

- vollständige bildgebende Dokumentation prä- und postinterventionell

\section{Ergebnis:}

3.1 Gesamtzahl/prozentuale Verteilung der verschiedenen Stentgrafts auf die jeweiligen Abschnitte der Aorta

3.2 Erfolgsdefinitionen:

- Erfolg = korrekte Platzierung des Stentgrafts, kein primäres Endoleak

- Teilerfolg = korrekte Platzierung des Stentgrafts mit primärem Endoleak Typ 2 und/oder 4

- Technischer Erfolg = Erfolg + Teilerfolg, da die Endoleaks Typ 2 und 4 unabhängig vom Prothesentyp und der prozeduralen Qualität der Intervention auftreten können

- Misserfolg = Fehlplatzierung, Endoleak Typ 1 oder 3 oder Abbruch der Intervention

3.3 Komplikationen:

Die Komplikationen werden nach Häufigkeit und Komplikationsgrad ausgewertet. Die Komplikationsgrade werden nach dem Schema der Society of Interventional Radiology (4) in Minor (A + B) und Major ( $\mathrm{C}-\mathrm{F}$ ) eingeteilt ( $\bullet$ Tab.4). Bei der Klassifikation F (Tod) wurde noch zwischen $<24 \mathrm{~h},<7 \mathrm{~d}$ und $<30$ Tagen differenziert. Hieraus werden Morbiditäts- und Mortalitätsraten und deren Ursachen bestimmt.

3.4 Strahlenexposition der Patienten:

Angaben zur Strahlenexposition gehören als wesentliches Qualitätsmerkmal auch zu den Pflichteingaben der Interventionsdatensätze. Registriert wurden die Durchleuchtungszeit und das Dosis-Flächen-Produkt.

\section{Ergebnisse}

\section{$\nabla$}

\section{Der DeGIR Datenpool}

Für das Jahr 2011 wurden bei 60021 Patienten 82881 Datensätze von interventionell radiologischen Eingriffen erfasst. Die Implantation von Stentgrafts in die verschiedenen Aortenabschnitte und in andere aneurysmatisch veränderte Arterien des Körperstamms und der Extremitäten nimmt mit 1653 Datensätzen Platz 9 in der Liste der 10 häufigsten Interventionen ein ( $\bullet$ Abb.1). Hierunter befanden sich 1167 EVAR-Interventionen, deren Analyse nachfolgend dargestellt wird.

\section{Patienten}

Behandelt wurden 993 Männer und 174 Frauen (Verhältnis 6:1) im Alter von 32 - 101 Jahren (Mittelwert 73,3 Jahre, Median 74 Jahre). - Abb. 2 gibt die Altersverteilung der Patienten wieder. Die Vertei- 

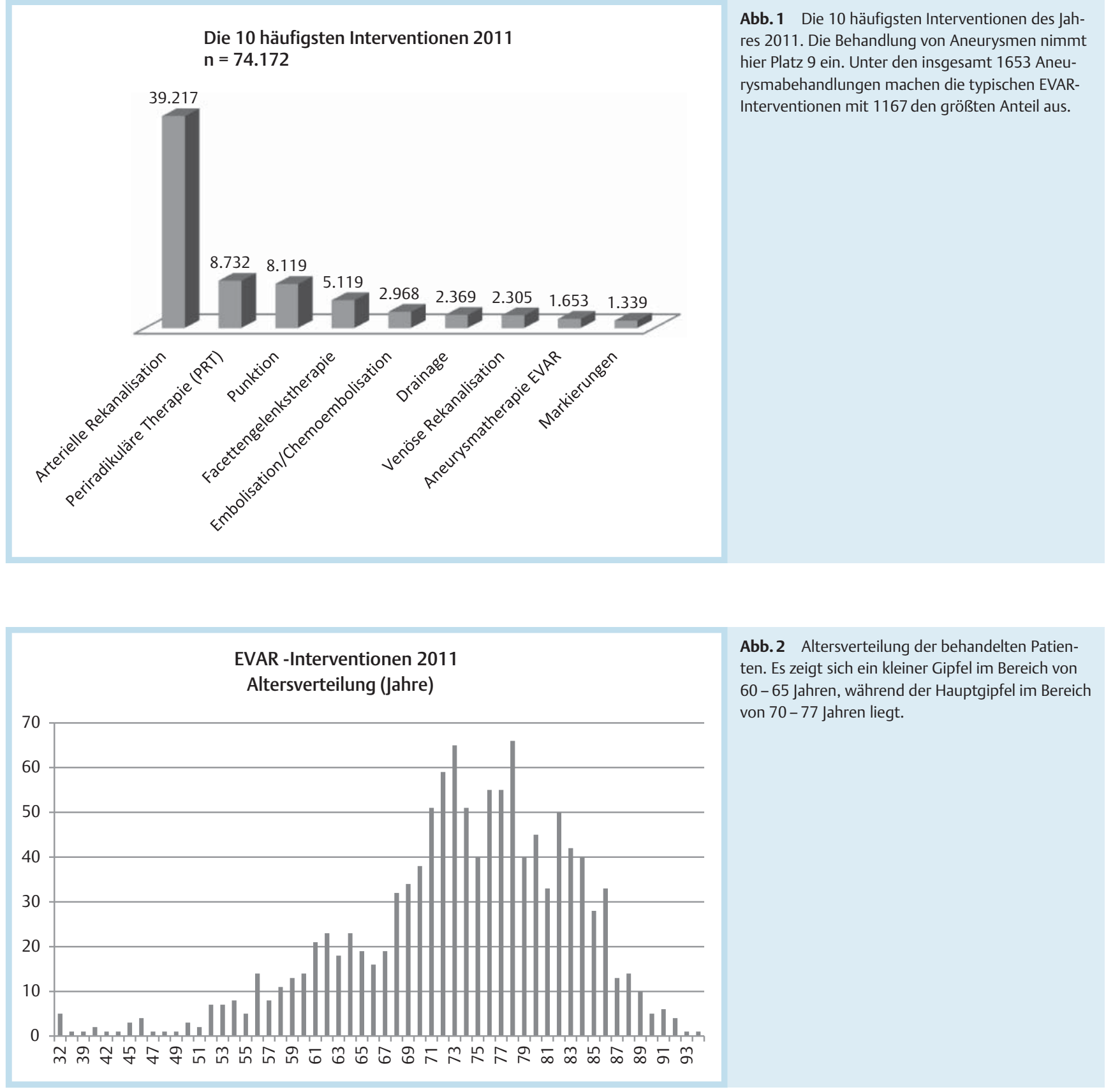

lungskurve zeigt einen kleinen Gipfel im Bereich von 60 - 65 Jahren, während der Hauptgipfel im Bereich von 70 - 77 Jahren liegt.

\section{Indikation}

Bei allen 1167 Patienten handelte es sich um Erst- und Einzelinterventionen. Notfallindikationen bestanden bei 145 Patienten $(12,4 \%)$; die übrigen 1022 Patienten (87,6\%) wurden elektiv behandelt.

Die interdisziplinäre Indikationsstellung ist ein wichtiges Qualitätsmerkmal. In 915 Datensätzen (78,4\%) ist eine gemeinsame Indikationsstellung mit mindestens einer weiteren Disziplin oder im Rahmen einer interdisziplinären Gefäßkonferenz angegeben. Bei 34 (2,9\%) Fällen wurde keine interdisziplinäre Indikationsstellung vermerkt und bei 218 (18,7\%) Datensätzen fehlen entsprechende Angaben. Hierbei ist zu bemerken, dass diese Angabe keine Pflichtangabe war. Es kann davon ausgegangen wer- den, dass ein wesentlicher Teil dieser Fälle ebenfalls interdisziplinär besprochen wurde.

\section{Lokalisation der Aneurysmen}

Die Lokalisation der Aneurysmen geht aus $\bullet$ Abb. 3 hervor. Danach betrafen $84,8 \%$ der Aneurysmen die Aorta abdominalis, davon waren $53,7 \%$ infrarenal lokalisiert und $12,0 \%$ in der thorakalen Aorta descendens. Die Aorta ascendens und der Arcus aortae wurden mit $0,7 \%$ respektive $2,5 \%$ angegeben; hierbei handelt es sich um Ausnahmeindikationen im Rahmen von Hybrideingriffen, die im Folgenden nicht weiter analysiert werden.

\section{Aneurysmatyp}

Eine Übersicht über die behandelten Aneurysmatypen ist in - Abb. 4 wiedergegeben. Hierzu konnten 881 Datensätze ausgewertet werden. Danach lagen in 79,4\% ein Aneurysma verum, 


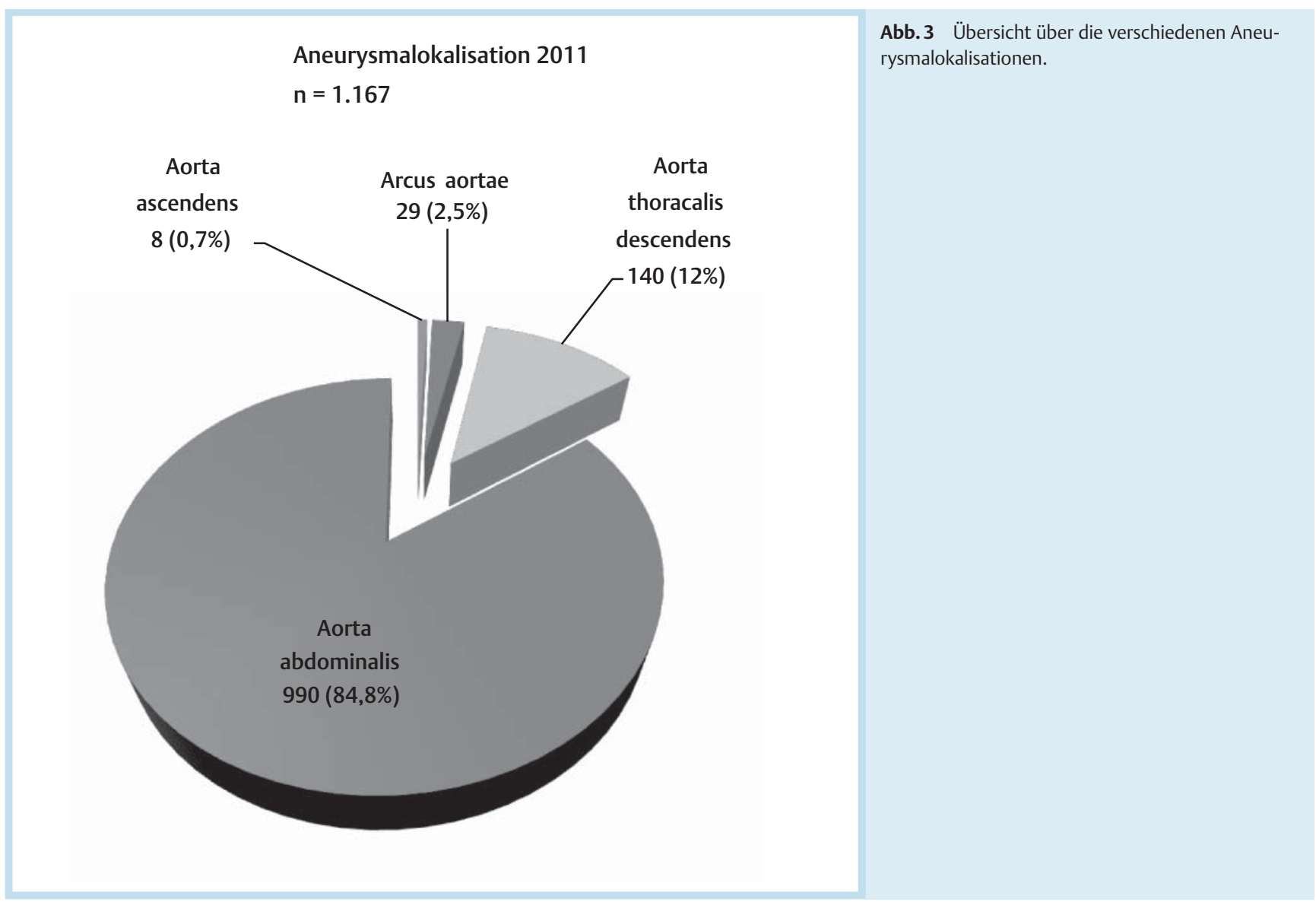

\begin{tabular}{|lrcl|}
\hline Kombination Prothesentypen & $\mathbf{n}$ & $\begin{array}{l}\text { Anteil der } \\
\text { Kombinationen }\end{array}$ & $\begin{array}{l}\text { Anteil aller } \\
\text { Interventionen }\end{array}$ \\
\hline Rohrprothesen + andere nicht spezifiziert & 7 & $12,5 \%$ & $0,7 \%$ \\
\hline fenestriert + andere nicht spezifiziert & 2 & $3,6 \%$ & $0,2 \%$ \\
\hline fenestriert + aorto-biiliakal & 20 & $35,7 \%$ & $2,1 \%$ \\
\hline fenestriert + aorto-biiliakal + andere nicht spezifiziert & 1 & $1,8 \%$ & $0,1 \%$ \\
\hline aorto-biiliakal + andere nicht spezifiziert & 8 & $14,3 \%$ & $0,8 \%$ \\
\hline aorto-biiliakal + Rohrprothesen & 14 & $25,0 \%$ & $1,5 \%$ \\
\hline aorto-biiliakal + Rohrprothesen + fenestriert & 1 & $1,8 \%$ & $0,1 \%$ \\
\hline aorto-biiliakal + aorto-monoiliakal & 2 & $3,6 \%$ & $0,2 \%$ \\
\hline aorto-monoiliakal + Rohrprothesen & 1 & $1,8 \%$ & $0,1 \%$ \\
\hline Summe & 56 & $100,0 \%$ & $5,9 \%$ \\
\hline
\end{tabular}

Tab. 1 Übersicht über die verwendeten Kombinationen von Prothesentypen. in 4,2\% ein Aneurysma spurium und in 6,5\% eine Dissektion der Aorta vor. Unter den komplizierten Formen war die gedeckte Perforation mit 7,5\% am häufigsten vertreten, während die Raten für freie Perforation 1,2\% sowie Behandlungen bei einem inflammatorischen bzw. mykotischen Aneurysma 1,0\% resp. 0,2\% betrugen.

\section{Aneurysmadurchmesser}

In 772 Fällen wurden Angaben zum Durchmesser des Aneurysmas gemacht. Der Median betrug für diese freiwillig übermittelten Daten 56,5 mm. Aneurysmen unter $50 \mathrm{~mm}$ Durchmesser kamen in dieser Gruppe nicht vor. 253 Fälle wurden bei einem Aneurysmadurchmesser zwischen 50 und $55 \mathrm{~mm}$ behandelt.

\section{Prozessdaten}

Implantierte Prothesentypen

- Abb. 5 gibt einen Überblick über die verwendeten Prothesentypen bei 940 Patienten. In mehr als der Hälfte der Fälle wurden aorto-biiliakale Stentgrafts eingesetzt, in knapp 20\% handelte es sich um Rohrprothesen und in 5,7\% um aorto-monoiliakale Grafts. Der Anteil der fenestrierten Prothesen betrug 3,2\%. In ebenfalls 3,2\% wurden andere im Abfragemenü nicht spezifizierte Stents verwendet. Kombinationen der verschiedenen Stenttypen wurden in 5,9\% verwendet. Tab. 1 gibt hierzu eine detaillierte Aufschlüsselung.

In der Gruppe der 77 rupturierten BAA wurden in 46 Fällen (59,7\%) Rohrprothesen, in 15 Fällen (19,5\%) aorto-biiliakale Prothesen und in 10 Fällen (13,0\%) aorto-monoiliakale Stentgrafts eingesetzt ( $\bullet$ Tab. 2). 


\section{Behandelter Aneurysmatyp 2011}

$\mathrm{n}=881$

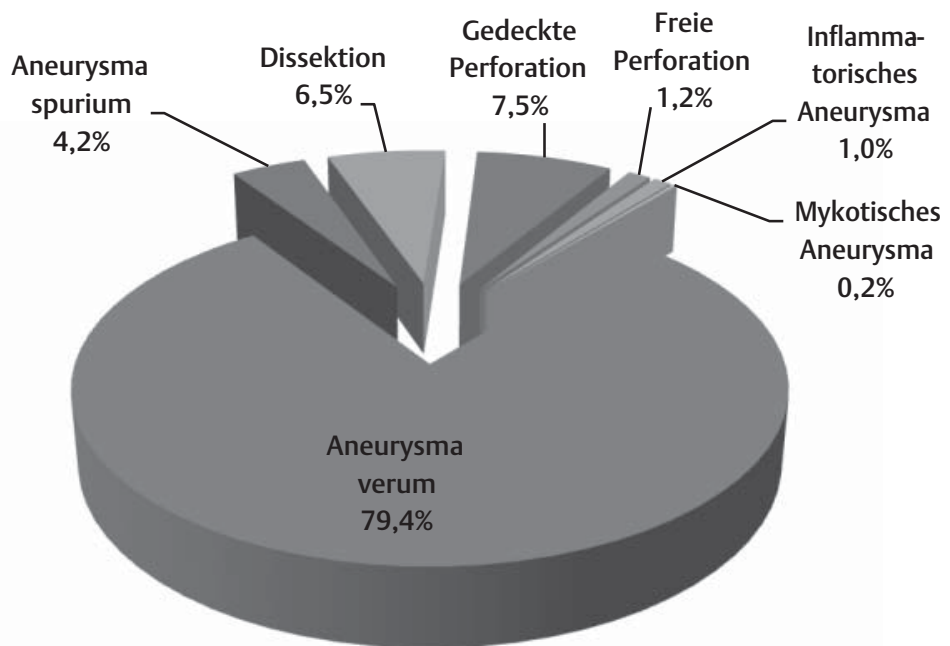

\begin{tabular}{|l|r|r|}
\hline \multicolumn{1}{|c|}{ Aneurysmatyp } & \multicolumn{1}{c|}{$\mathbf{n}$} & \multicolumn{1}{c|}{ Anteil } \\
\hline Aneurysma verum & 700 & $79,5 \%$ \\
\hline Aneurysma spurium & 36 & $4,1 \%$ \\
\hline Dissektion & 57 & $6,5 \%$ \\
\hline Gedeckte Perforation & 66 & $7,5 \%$ \\
\hline Freie Perforation & 11 & $1,2 \%$ \\
\hline Inflammation & 9 & $1,0 \%$ \\
\hline Infektion(mykotisches Aneurysma) & 2 & $0,2 \%$ \\
\hline Summe & 881 & $\mathbf{1 0 0 , 0 \%}$ \\
\hline
\end{tabular}

Abb.4 Übersicht über die behandelten Aneurysmatypen.
Tab. 2 Übersicht über die verwendeten Prothesentypen bei rupturierten Aortenaneurysmen.

\begin{tabular}{|c|c|c|}
\hline Stentgrafttyp bei rupturierten Aneurysmen & $\mathbf{n}$ & $\%$ \\
\hline Rohrprothesen & 46 & $59,7 \%$ \\
\hline aorto-biiliakal + Rohrprothesen & 1 & $1,3 \%$ \\
\hline aorto-biiliakal & 15 & $19,5 \%$ \\
\hline aorto-monoiliakal & 10 & $13,0 \%$ \\
\hline fenestriert & 0 & $0,0 \%$ \\
\hline andere nicht spez. & 3 & $3,9 \%$ \\
\hline ohne Angabe & 2 & $2,6 \%$ \\
\hline Summe & 77 & $100,0 \%$ \\
\hline
\end{tabular}

Technischer Erfolg, Endoleaks (= Teilerfolg)

und Misserfolg

In $\odot$ Abb. 6 sind die Raten für Erfolg, Teilerfolg und Misserfolg grafisch und tabellarisch - nach den jeweiligen StentgraftTypen aufgeschlüsselt - dargestellt. Die Erfolgsrate für alle Interventionen liegt bei 86,1\%, die Rate an primären Endoleaks Typ 2 oder Typ 4 (=Teilerfolg) bei 8,5\%.

Da Endoleaks Typ 2 und 4 nicht prozessassoziiert sind, wurden sie per definitionem in die Gruppe des „Technischen Erfolgs“ mitaufgenommen. $\bullet$ Abb. 6 gibt die Raten des Technischen Erfolgs für die einzelnen Prothesentypen wieder. Die Gesamtrate des Technischen Erfolgs beträgt 94,6\% und somit der Anteil der Therapieversager 5,4\%. Bezogen auf die einzelnen Prothesentypen ergibt sich die höchste Rate des technischen Erfolgs für die aorto-biiliakalen Prothesen (95,8\%), während bei der Kombination verschiedener Endoprothesentypen die entsprechende Rate mit $89,3 \%$ deutlich niedriger lag.

\section{Analyse der Endoleaks}

- Tab. 3 zeigt Anteil, Typ und Verteilung der Endoleaks bezogen auf die verschiedenen Stentgraft-Typen bei 944 Datensätzen. Zwei Drittel aller Endoleaks waren dem Typ 2 zuzuordnen; knapp ein Drittel der Endoleaks entsprach einem Typ 1. Bezogen auf die Gesamtzahl der Interventionen lag bei $4 \%$ ein Typ-1-Leck vor. Endoleaktypen 3 und 4 ereigneten sich mit 3,3 bzw. 0,8\%. In absoluten Zahlen traten diese Endoleaks lediglich in 5 Fällen auf. Bezogen auf alle Interventionen ergaben sich Raten für den Endoleaktyp 1 von gut 3,9\% und für den Endoleaktyp 2 von 8,4\%. Die Typen 3 und 4 lagen unter 0,5\%. In der Detailanalyse bezogen auf die verwendeten Stentgraft-Typen ergab sich bei Rohrprothesen die niedrigste $(7,5 \%)$ und bei der Kombination verschiedener Stentgraft-Typen die höchste (17,9\%) Endoleakrate als Anteil an allen Leaks. Bei den übrigen Stentgraft-Typen bewegte sich der Anteil an Endoleaks zwischen 10\% und 14,4\%. 
Implantierte Stentgraft-Typen 2011

$\mathrm{n}=940$

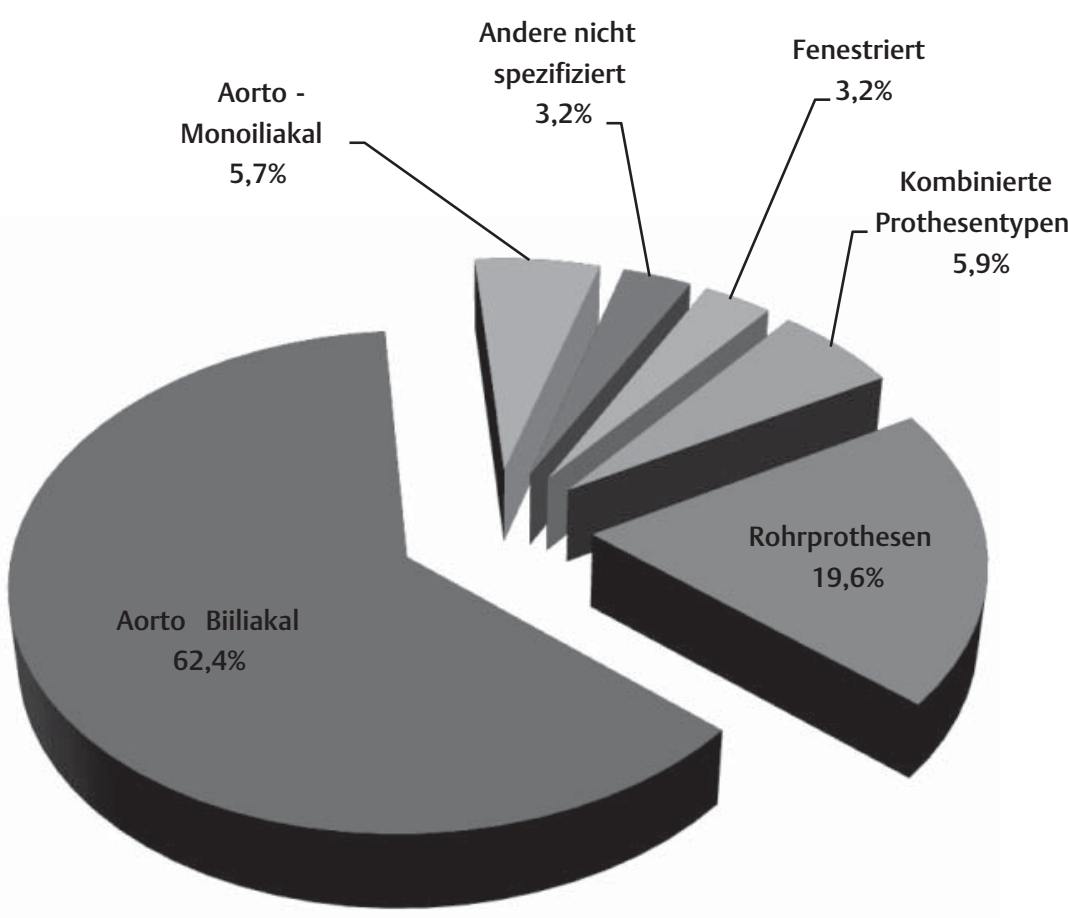

Abb.5 Übersicht über die verwendeten Prothesentypen. Die kombinierten Prothesentypen sind in Tab. 1 genauer aufgeführt.

Tab. 3 Anzahl, Art und prozentualer Anteil der Endoleaktypen, bezogen einmal auf die Gruppe aller Endoleaks und einmal auf das Gesamtkollektiv aller EVAR.

\begin{tabular}{|c|c|c|c|c|c|c|c|c|c|c|c|c|c|}
\hline \multicolumn{2}{|l|}{ Primäres Endoleak } & \multicolumn{3}{|c|}{ Typ 1} & \multicolumn{3}{|c|}{ Typ 2} & \multicolumn{3}{|c|}{ Typ 3} & \multicolumn{3}{|c|}{ Typ 4} \\
\hline Stentgraft-Typ & $\mathbf{n}$ & $\mathbf{n}$ & $\begin{array}{l}\text { Anteil } \\
\text { Endoleaks }\end{array}$ & $\begin{array}{l}\text { Anteil } \\
\text { gesamt }\end{array}$ & $\mathbf{n}$ & $\begin{array}{l}\text { Anteil } \\
\text { Endoleaks }\end{array}$ & $\begin{array}{l}\text { Anteil } \\
\text { gesamt }\end{array}$ & $\mathbf{n}$ & $\begin{array}{l}\text { Anteil } \\
\text { Endoleaks }\end{array}$ & $\begin{array}{l}\text { Anteil } \\
\text { gesamt }\end{array}$ & $\mathbf{n}$ & $\begin{array}{l}\text { Anteil } \\
\text { Endoleaks }\end{array}$ & $\begin{array}{l}\text { Anteil } \\
\text { gesamt }\end{array}$ \\
\hline Rohr & 14 & 9 & $4,8 \%$ & $0,96 \%$ & 5 & 2,7 & $0,53 \%$ & 0 & 0 & $0,00 \%$ & 0 & 0 & $0,00 \%$ \\
\hline aorto-biiliakal & 85 & 18 & $3,1 \%$ & $1,91 \%$ & 63 & 10,7 & $6,70 \%$ & 3 & 0,5 & $0,32 \%$ & 1 & 0,2 & $0,11 \%$ \\
\hline aorto-monoiliakal & 5 & 2 & $4,2 \%$ & $0,21 \%$ & 3 & 6,3 & $0,32 \%$ & 0 & 0 & $0,00 \%$ & 0 & 0 & $0,00 \%$ \\
\hline $\begin{array}{l}\text { andere nicht } \\
\text { spezifiziert }\end{array}$ & 4 & 1 & $3,2 \%$ & $0,11 \%$ & 3 & 9,7 & $0,32 \%$ & 0 & 0 & $0,00 \%$ & 0 & 0 & $0,00 \%$ \\
\hline fenestriert & 3 & 2 & $6,7 \%$ & $0,21 \%$ & 1 & 3,3 & $0,11 \%$ & 0 & 0 & $0,00 \%$ & 0 & 0 & $0,00 \%$ \\
\hline $\begin{array}{l}\text { kombinierte } \\
\text { Stentgraft-Typen }\end{array}$ & 10 & 5 & $8,9 \%$ & $0,53 \%$ & 4 & 7,1 & $0,43 \%$ & 1 & 1,8 & $0,11 \%$ & 0 & 0 & $0,00 \%$ \\
\hline Summe & 121 & 37 & $30,9 \%$ & $3,94 \%$ & 79 & $65,3 \%$ & $8,40 \%$ & 4 & $3,3 \%$ & $0,43 \%$ & 1 & 0,8 & $0,11 \%$ \\
\hline
\end{tabular}

\section{Komplikationen}

Anteil und Schweregrad nach SIR, sekundäre Not-Operation

Bei 48 (4\%) der 1.167 behandelten Patienten traten Komplikationen auf. Für die Stratifizierung der Komplikationsgrade wurde die Einteilung der Society of Interventional Radiology (SIR) verwendet ( $\bullet$ Tab.4). Es fällt auf, dass der Anteil der Major-Komplikationen (Grad D - F) an den 48 Komplikationen etwas über $60 \%$ liegt. Bezogen auf alle behandelten Patienten liegt er jedoch mit $2,5 \%$ in einem erfreulich niedrigen Bereich.

Bei 5 von 1167 (0,43\%) Patienten erfolgte eine Konversion zur klassischen offenen Operation.

\section{Ursachen der Komplikationen und Verlauf}

- Tab. 5 gibt eine Aufschlüsselung nach den Ursachen der Komplikationen wieder. Danach liegen arterielle Blutung und arterieller Gefäßverschluss mit jeweils 16,7\% an erster Stelle gefolgt von Fehllagen der implantierten Stentgrafts und Organminderfunktion/-versagen mit 12,5\% und 10,4\%. Die übrigen Komplikationsarten liegen im einstelligen prozentualen Bereich. Bei 10 von 48 Komplikationen (20,8\%) wurde als Ursache der Begriff „Sonstige“ kodiert. Leider sieht die Software keine Möglichkeit der Freitexteingabe und folglich auch keine weitere Aufschlüsselung vor. In einer weiteren Abfrage wurden die Angaben „Sonstige“ mit den Angaben „Interventionsabbruch“, „sekundäre Not-OP“ und „Prothesenfehllage“ verknüpft. Hierbei konnte ermittelt werden, dass 


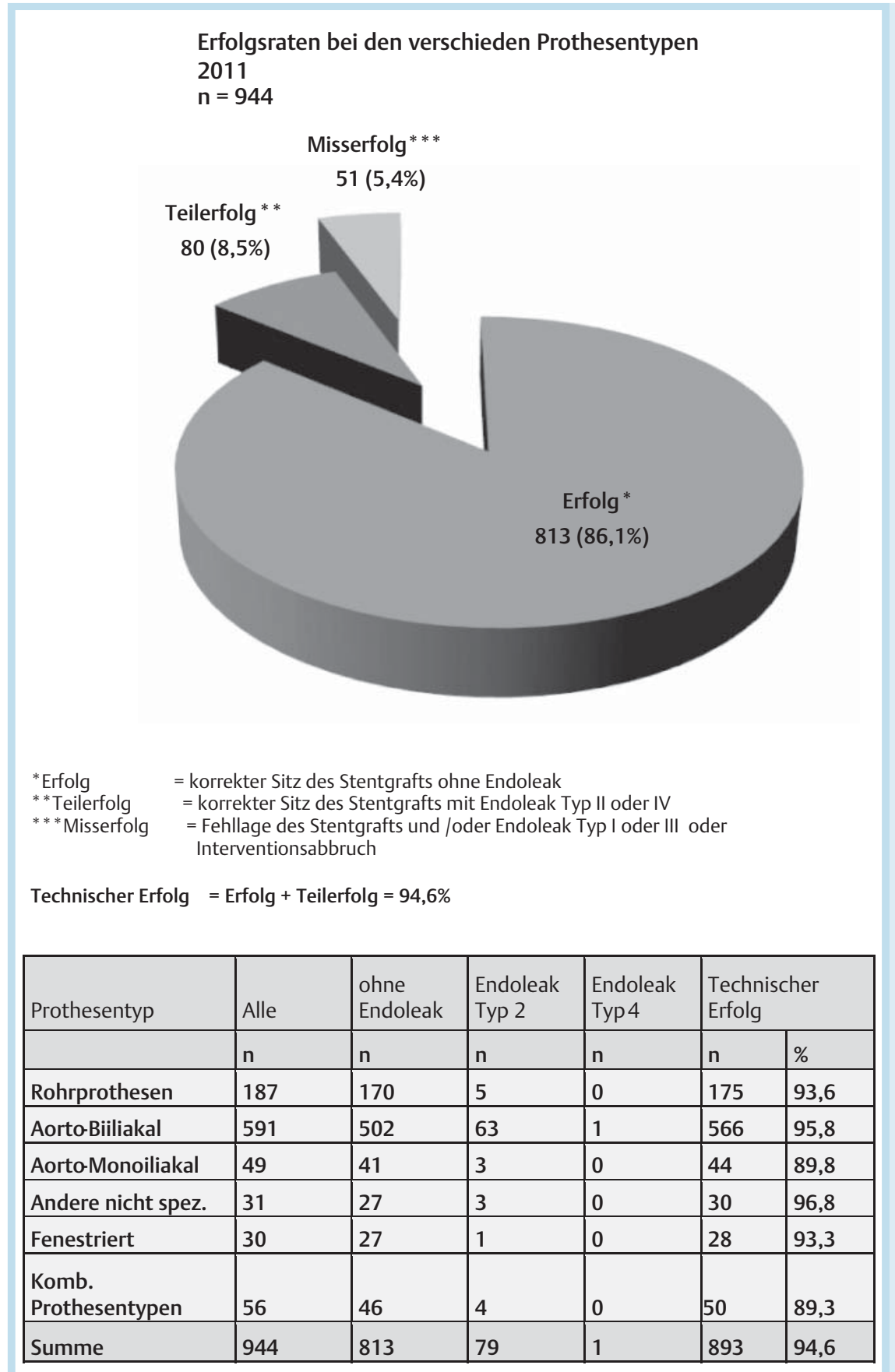

Abb.6 Übersicht über Erfolg, Teilerfolg und Misserfolg bei EVAR-Interventionen (Grafik). Der technische Erfolg fasst die Gruppen ohne Endoleak und mit Endoleak Typ 2 und 4 zusammen (Tabelle).

\begin{tabular}{|lccc|}
\hline Komplikationsgrad nach SIR & Anzahl & Anteil Komplik. & Anteil gesamt \\
\hline A - kein Therapiebedarf, keine Konsequenzen & 8 & $17,0 \%$ & $0,69 \%$ \\
\hline B - symptomatische Behandlung, ggf. Observation über Nacht & 4 & $8,5 \%$ & $0,34 \%$ \\
\hline C- Therapiebedarf, kurze Hospitalisation (<48 h) & 6 & $12,8 \%$ & $0,51 \%$ \\
\hline $\begin{array}{l}\text { D - höherer Therapiebedarf, ungeplanter Anstieg des Behandlungs- } \\
\text { levels, verlängerte Hospitalisation (>48 h) }\end{array}$ & 15 & $31,9 \%$ & $1,29 \%$ \\
\hline E - Dauerschäden & 1 & $2,1 \%$ & $0,09 \%$ \\
\hline F- Tod & 13 & $27,7 \%$ & $1,11 \%$ \\
\hline Summe & 47 & $100,0 \%$ & $4,03 \%$ \\
\hline
\end{tabular}

Tab. 4 Übersicht über die Schweregrade der Komplikationen und deren jeweiligen Anteil. 


\begin{tabular}{|c|c|c|c|}
\hline Komplikation & $\mathbf{n}$ & Anteil an allen Komplikationen & Anteil am gesamten Kollektiv \\
\hline Aneurysma an der Punktionsstelle & 2 & $4,2 \%$ & $0,17 \%$ \\
\hline arterielle Blutung & 8 & $16,7 \%$ & $0,69 \%$ \\
\hline arterieller Gefäßverschluss & 8 & $16,7 \%$ & $0,69 \%$ \\
\hline Infektion/Abszess & 4 & $8,3 \%$ & $0,34 \%$ \\
\hline kardiale Komplikation & 3 & $6,2 \%$ & $0,26 \%$ \\
\hline $\begin{array}{l}\text { Prothesenfehllage, primär } \\
\text { oder sekundär }\end{array}$ & 6 & $12,5 \%$ & $0,51 \%$ \\
\hline Organminderfunktion/-versagen & 5 & $10,4 \%$ & $0,43 \%$ \\
\hline Parenchymischämie oder -infarkt & 1 & $2,1 \%$ & $0,09 \%$ \\
\hline pulmonale Komplikation & 1 & $2,1 \%$ & $0,09 \%$ \\
\hline sonstige & 10 & $20,8 \%$ & $1,17 \%$ \\
\hline Summe & 48 & $100,0 \%$ & $4,11 \%$ \\
\hline
\end{tabular}

Tab. 5 Übersicht über Art, Anzahl und prozentualen Anteil der Komplikationen, bezogen einmal auf die Gruppe aller Komplikationen und einmal auf das Gesamtkollektiv aller EVAR.

\begin{tabular}{|c|c|c|c|c|c|}
\hline Komplikationsgrad nach SIR & $\mathbf{n}$ & $\begin{array}{l}\text { selbst } \\
\text { behandelt }\end{array}$ & $\begin{array}{l}\text { behandelt durch } \\
\text { andere Disziplin }\end{array}$ & $\begin{array}{l}\text { erfolgreich } \\
\text { behandelt }\end{array}$ & $\begin{array}{l}\text { nicht } \\
\text { behandelt }\end{array}$ \\
\hline A - kein Therapiebedarf & 8 & - & - & - & 8 \\
\hline B - symptomatische Behandlung..... & 4 & 0 & 4 & 4 & 0 \\
\hline C - Therapiebedarf, kurze Hosp. ( $<48$ h) & 7 & 4 & 3 & 6 & 0 \\
\hline D - höherer Th.-Bed. Hosp. (> 48 h) & 15 & 4 & 8 & 10 & 3 \\
\hline E-Dauerschäden & 1 & 1 & 0 & 0 & 0 \\
\hline $\mathrm{F}-\mathrm{Tod}$ & 13 & 1 & 4 & 0 & 8 \\
\hline Summe & 48 & 10 & 19 & 20 & 19 \\
\hline
\end{tabular}

Tab. 6 Übersicht über das Schicksal der Patienten mit Komplikationen.

\section{Interventionen mit tödlichem Ausgang \\ $\mathrm{n}=13$}

Abb. 7 Zeitlicher Zusammenhang zwischen der Intervention und dem Tod der Patienten.

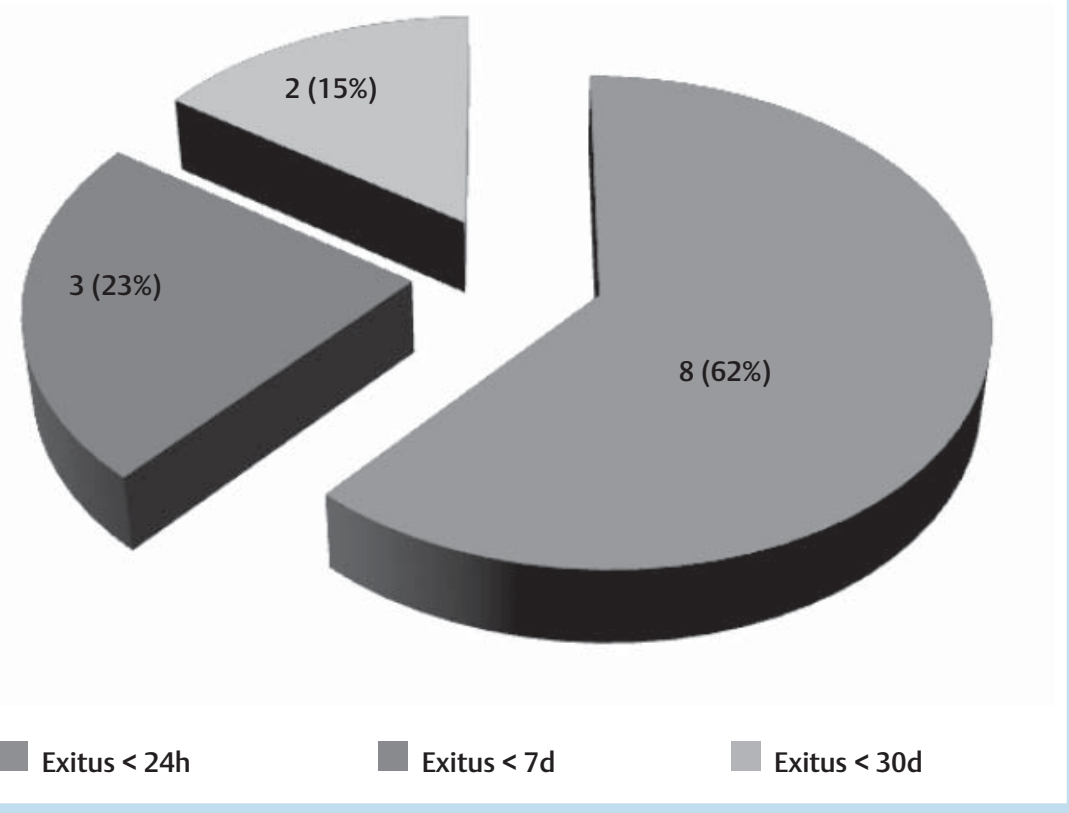

in 9 dieser Fälle eine Prothesenfehllage bestand. Ein Interventionsabbruch oder eine sekundäre Not-Operation wurden nicht angegeben.

In $\bullet$ Tab. 6 ist der Verlauf der Komplikationen wiedergegeben. 33 von 47 Komplikationen (70,2\%) wurden mit einer Sekundärbehandlung, 13 radiologisch interventionell, 20 von einer anderen Disziplin versorgt. In 25 Fällen war die Behandlung der jeweiligen Komplikation erfolgreich (12 radiologisch interventionell und 13 durch andere Disziplinen).

\section{Letalität}

In 13 Fällen führten die Komplikationen zum Tode. Insgesamt ergibt sich eine Letalität von $1,11 \%$ ( $\bullet$ Tab. 4 ).

Acht Patienten verstarben innerhalb der ersten $24 \mathrm{~h}$ nach dem Eingriff, 3 innerhalb der ersten 7 und 2 innerhalb der ersten 30 Tage ( $\bullet$ Abb. 7). $\bullet$ Tab. 8 gibt einen Überblick über die Todesursachen. In 4 Fällen wurde der Begriff „Sonstige“ kodiert. Da die Software hier, wie bereits oben ausgeführt, keinen Freitext zulässt, existieren hier keine weiteren Angaben. Eine kombinierte 
Tab. 7 Komplikationshäufigkeit und Komplikationsgrad bei rupturierten Aneurysmen.

\begin{tabular}{|lrc|}
$\begin{array}{l}\text { Komplikationen bei } \\
\text { rupturierten Aneurysmen }\end{array}$ & n & $\begin{array}{l}\text { Anteil rupturierter } \\
\text { Aneurysmen }\end{array}$ \\
\hline keine & 63 & $84,0 \%$ \\
\hline Komplikation Grad B & 1 & $1,3 \%$ \\
\hline Komplikation Grad D & 2 & $2,7 \%$ \\
\hline Komplikation Grad F (Tod) & 9 & $12,0 \%$ \\
\hline Summe & 75 & $100,0 \%$ \\
\hline
\end{tabular}

Tab. 8 Übersicht über die Ursachen der Fälle mit tödlichem Ausgang.

\begin{tabular}{|lc|}
\hline Todesursache & $\mathbf{n}$ \\
\hline Organminderfunktion/-versagen & 3 \\
\hline arterielle Blutung & 3 \\
\hline kardiale Komplikation & 3 \\
\hline sonstige (Prothesenfehllage $n=3)$ & 4 \\
\hline Summe & 13 \\
\hline
\end{tabular}

Datenabfrage ergab eine Prothesenfehllage in 3 dieser Fälle, eine sekundäre Not-Operation wurde bei keinem dieser Patienten durchgeführt.

Die aufgezählten Komplikationen umfassen die Gruppe aller EVAR einschließlich der Behandlungen wegen eines rupturierten Aneurysmas. Betrachtet man die Gruppe der rupturierten Aneurysmen getrennt ( $\bullet$ Tab.7), so finden sich viele der erwähnten Komplikationen in dieser Gruppe. Insbesondere sind die 9 Todesfälle in dieser Gruppe zu erwähnen. Unter Berücksichtigung dieser Fälle kann für die elektiven Eingriffe eine Letalität von 0,34\% errechnet werden. Auch die Letalität für rupturierte Aneurysmen ist in Anbetracht der Schwere der akuten Erkrankung mit $12 \%$ als sehr niedrig zu betrachten.

\section{Daten zur Strahlenexposition}

Zum Dosisflächenprodukt und zur Durchleuchtungszeit wurden nur teilweise verwertbare Daten kodiert. Daher ist hier nur der Median als repräsentative Größe verwertbar. Nach Auswertung von 797/838 verwertbaren Datensätzen für Flächendosisprodukt/Durchleuchtungszeit ergab sich für das Flächendosisprodukt ein Median von 10676,5 cGy× $\mathrm{cm}^{2}$ und für die Durchleuchtungszeit ein Median von 17,32 min.

\section{Diskussion}

Die Anzahl an endovaskulären Behandlungen von Aortenaneurysmen ist in den letzten Jahren stetig gestiegen. Nach den Angaben des statistischen Bundesamts kommen jährlich pro 100000 Einwohner 74,5 männliche und 9,8 weiblich Patienten zu einer elektiv endovaskulären Behandlung. 12,5 männliche und 2,6 weiblichen Patienten (pro 100000 Einw.) werden mit einem rupturierten Bauchaortenaneurysma stationär aufgenommen [5]. Die Zahl der endovaskulären Interventionen von abdominalen Aortenaneurysmen stieg bei Männern von n=2909 im Jahr 2006 auf $\mathrm{n}=5257$ im Jahr 2009, während bei Frauen die Zahl der registrierten Eingriffe von 346 im gleichen Zeitraum auf 670 anwuchs. Die Ursache hierfür ist nicht alleine in einer Zunahme der Inzidenz von Aneurysmen zu sehen. Vielmehr hat die technische Weiterentwicklung und Verbesserung der Stentgrafts und ihrer
Applikationssysteme zu einer Verschiebung der Behandlungsmethode von der klassischen Operation hin zur EVAR-Intervention geführt. Selbst stark elongiert und gewunden verlaufende Beckenarterien können mit speziellen Drahttechniken für die Passage der zunehmend kleinen und flexiblen Prothesenkörper begradigt werden [6].

Das Ziel der vorliegenden Arbeit ist allein die Bestimmung der Behandlungsqualität anhand vorgegebener Kriterien für Indikation, Prozessdaten sowie den technischen Erfolg und die mit dem Eingriff verbundenen Komplikationen.

\section{Indikation}

Erfasst wurden alle Arten von Aneurysmen bzw. Erkrankungen der Aorta in allen Gefäßabschnitten. Erwartungsgemäß waren das Aneurysma verum mit über $79 \%$ und die Aortenabschnitte IV und V mit zusammen $84 \%$ die mit Abstand häufigste Indikation.

Das Verhältnis 85,6\% für elektive Eingriffe zu 12,4\% für Notfallindikationen entspricht der Größenordnung, wie sie auch in offiziellen Statistiken dargestellt wird [5].

Ein wichtiges Merkmal der Prozessqualität ist die interdisziplinäre Indikationsstellung. Der hier ermittelte Anteil von $78,4 \%$ ist nicht befriedigend. In 18,7\% fehlen allerdings zu diesem Punkt explizite Angaben. Die Erklärung dürfte darin liegen, dass für dieses Kriterium keine Pflichteingabe vorgesehen war und viele diesen Punkt als Selbstverständlichkeit ansehen. Dies erklärt sich aus der routinemäßigen Zuweisung von Patienten mit Aneurysmen durch andere medizinische Disziplinen. Geht man davon aus, dass diese Patienten gefäßchirurgisch aufgenommen und betreut werden und dass die meisten Eingriffe ohnehin interdisziplinär oder zumindest mit gefäßchirurgischer Begleitung durchgeführt werden, dürfte der Anteil interdisziplinär besprochener Fälle bei über $95 \%$ liegen. Die Rate an rupturierten Aneurysmen lag mit knapp 9\% deutlich niedriger als die Inzidenz in der deutschlandweiten Erfassung von Santosa et al. [5]. Hier wurden 14,5 \% für Männer und 21,5\% für Frauen bezogen auf das Jahr 2009 angegeben. Da die Notfallversorgung häufig außerhalb der normalen Dienstzeiten erfolgt, kann angenommen werden, dass in der geführten Datenbank zu dieser Indikation nur unvollständige Daten vorliegen.

\section{Verwendete Stentgraft-Typen}

Aorto-biiliakale Prothesen (62,4\%) und Rohrprothesen (19,6\%) waren die mit Abstand am häufigsten verwendeten Stentgrafts, was durch die Häufigkeit der Aneurysmatypen und deren Lokalisationen vorgegeben war. Der Anteil der übrigen Prothesentypen und der Kombinationen liegt deutlich unter 10\%.

\section{Erfolg, Teilerfolg, Misserfolg}

Für alle Stentprothesen zusammen errechnete sich eine Erfolgs-

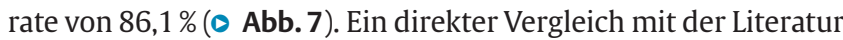
ist in dieser Form nicht möglich, da wir in unserer Auswertung auch die Notfalleingriffe bei rupturierten Aneurysmen miteingeschlossen haben.

Typ-2- und Typ-4-Endoleaks sind von der Eingriffsqualität unabhängig. Daher kann man vom klinischen Standpunkt diese Leckageformen [7], bei uns als Teilerfolg beschrieben, mit der Gruppe der erfolgreichen Prozeduren zusammenfassen. Da dies in der Literatur auch getan wird, lässt sich auf diese Weise Vergleichbarkeit herstellen. Es werden primäre Erfolgsraten von 98 - $100 \%$ bei rein elektiven Eingriffen angegeben [7, 8]. Berücksichtigt man den Anteil von knapp $11 \%$ an komplikativen Aneurysmen in un- 
serem Patientengut, so liegt die Erfolgsrate bei unseren Daten von $94,6 \%$ in einem exzellenten Bereich.

Betrachtet man in unserem Datengut sämtliche Endoleaks unabhängig vom Typ, so ergibt sich eine primäre Endoleakrate von $12,3 \%$. Berücksichtigt man, dass in der Literatur Endoleakraten von $16-30 \%$ angegeben werden [9-11], liegt die Rate mit 12,3\% erfreulich niedrig. Noch niedriger ist die Rate an primären Typ-1und Typ-3-Endoleaks mit 4,37\%. Dies ist besonders relevant, da es sich hierbei um die Fälle handelt, welche mithilfe der EVAR nicht ausreichend behandelt worden sind.

Das Typ-2-Endoleack war mit einem Anteil von zwei Dritteln aller Endoleaks der bei Weitem häufigste Typ ( $\bullet$ Tab. 3). Mit 8,4\% aller Intervention liegt auch hier die Rate eher niedrig im Vergleich mit der Literatur [12]. Das zeitnah behandlungsbedürftige Endoleak Typ 1 wurde in knapp einem Drittel aller Endoleaks registriert. Sein Anteil an allen Interventionen betrug lediglich 3,3\%, was ebenfalls im Vergleich mit der Literatur [12] niedrig ist. Sicher haben die Aneurysmakonfiguration und der Halswinkel einen wesentlichen Einfluss auf die Entstehung eines primären Endoleaks Typ 1 (13). Endoleaks Typ 3 wurden nur in 4 Fällen und Typ 4 nur in einem Fall gemeldet.

Bei insgesamt 11 (1,2\%) der behandelten Patienten war die Intervention nicht erfolgreich, d.h. es konnte kein korrekter Sitz des Stentgrafts erzielt werden. In 5 Fällen erfolgte eine Konversion zu einer offenen Operation.

\section{Morbidität und Mortalität}

Abgesehen von den Endoleaks, die gesondert analysiert wurden, ergaben sich im Gesamtkollektiv bei 48 (4,03\%) Patienten Komplikationen, wobei es sich in fast zwei Dritteln um Major-Komplikationen nach SIR [4] handelt. Dies macht die Schwere der Aneurysmaerkrankung und das spezifische Risiko der Therapie deutlich. Abgesehen von eingriffsbedingten Komplikationen wie arteriellen Blutungen, Gefäßverschlüssen, Fehllagen des Stentgrafts und Infektionen, die mehr als die Hälfte aller Komplikationen ausmachen ( $\bullet$ Tab.4, 5), ergibt sich auch eine hohe Komorbiditätsrate. Die Behandlung der Komplikationen erfolgte in $27,6 \%$ durch den interventionell tätigen Radiologen selbst und in 42,6\% durch eine andere Disziplin (Chirurgie/Gefäßchirurgie). In 53,2\% war diese Therapie erfolgreich ( $\bullet$ Tab. 6 ).

Infektionen machen mit 8 Fällen und einer Rate von 8,3\% der Komplikationen respektive von $0,3 \%$ am Gesamtkollektiv nur einen geringen Anteil aus und wurden daher keiner weiteren Analyse unterzogen. Hahne et al. [14] konnten zeigen, dass im Gegensatz zu der bisher meist durchgeführten Operation mit Explantation der Prothese, Debridement und extraanatomischem Bypass auch eine konservative antibiotische Therapie erfolgreich sein kann.

Die rupturierten Aneurysmen wurden einer gesonderten Analyse unterzogen ( $\bullet$ Tab.7). Es ist sinnvoll, diese Tabelle mit der - Tab. 6 zu vergleichen, um das Auftreten von Komplikationen bei rupturierten Aneurysmen zu erfassen. Nachfolgend wird eine Aufschlüsselung nach Komplikationsgraden gegeben:

\section{Komplikation Grad A:}

Von den 8 Fällen betraf keiner ein rupturiertes Aneurysma.

Komplikation Grad B:

Einer (25\%) der 4 in $\bullet$ Tab. 6 aufgeführten Fälle entfiel auf ein rupturiertes Aneurysma.

Komplikation Grad C:

Von den 7 Fällen der $\bullet$ Tab. 6 betraf keiner ein rupturiertes Aneurysma.

\section{Komplikation Grad D:}

Zwei (13,3\%) von insgesamt 7 Fällen entfielen auf rupturierte Aneurysmen.

\section{Komplikation Grad E:}

Der eine in $\bullet$ Tab. 6 aufgeführte Fall betraf kein rupturiertes Aneurysma.

\section{Komplikation Grad F:}

Hier entfielen 9 (69,2\%) von 13 Todesfällen auf rupturierte Aneurysmen.

Bei rupturierten Aneurysmen betrugen die Komplikationshäufigkeit $16 \%$ und die Letalität $12 \%$.

Die Eingriffsletalität des Gesamtkollektivs liegt mit 1,11\% vergleichsweise niedrig $[12,13]$. Auch hier muss berücksichtigt werden, dass nicht zwischen elektiven und Noteingriffen unterschieden wird. Betrachtet man nur die elektiven EVAR, so liegt die Letalität dieser Eingriffe mit 0,34\% nochmals deutlich niedriger und auch unter den Literaturangaben. In den bereits zitierten $\mathrm{Ar}$ beiten von van Keulen et al. [7] und Pitton et al. [8] ist die Eingriffsletalität mit 0,8 resp. 2 \% für elektive Prozeduren angegeben. In einer von Rayt et al. 2008 [15] publizierten Metaanalyse von 31 Studien mit 982 Interventionen bei rupturierten Bauchaortenaneurysmen betrug die Letalität in dieser Notfallgruppe noch $24 \%$ und war damit doppelt so hoch wie in unserem Patientengut. Möglicherweise ist die günstige Rate in unserem Krankengut durch die vergleichsweise geringe Zahl rupturierter Aneurysmen mit eher günstigem Behandlungsergebnis bedingt.

Die Analyse der Todesursachen ergab neben Organversagen $(\mathrm{n}=3)$, arteriellen Blutungen $(\mathrm{n}=3)$ und kardialen Komplikationen $(n=3)$ auch 4 mit dem Begriff „Sonstige“ kodierte Komplikationen. Die Erfassungssoftware lässt an dieser Stelle keinen Freitext zu, was für die Zukunft geändert werden soll. Weitere Analysen mit verknüpften Abfragen der Datenbank ergaben, dass in 3 dieser Fälle Fehllagen der Stentgrafts bestanden. Eine sekundäre Not-Operation (Konversion) wurde in keinem dieser Fälle durchgeführt.

\section{Daten zur Strahlenexposition}

Sowohl die Eingaben zur Durchleuchtungszeit als auch zum Dosis-Flächen-Produkt sind von den Nutzern der Software kritisiert worden. Die Gründe liegen hauptsächlich in den unterschiedlichen Größenordnungen für das Dosisflächenprodukt, die von den verschiedenen Angiografiesystemen ausgegeben werden. Diese müssen dann jeweils aufwendig in die von der Software geforderte einheitliche Größenordnung umgerechnet werden. Als Konsequenz aus diesem Problem wird die Dateneingabe so modifiziert werden, dass aus den 2 gängigsten Größeneinheiten gewählt werden kann. Der mediane Expositionswert liegt unter $10700 \mathrm{cGy} \times \mathrm{cm}^{2}$ und kann im Hinblick auf die Komplexität der Eingriffe als vertretbar angesehen werden. Gleiches gilt auch für die mediane Durchleuchtungszeit von 17,32 min. Beide Werte können als Orientierung für weitere Analysen dienen.

\section{Schlussfolgerungen}

Die vorliegende Analyse ist die erste Jahresauswertung eines neu geschaffenen Erfassungssystems der DeGIR für EVAR-Interventionen.

Die mit diesem System erfassten EVAR-Interventionen erlauben hinsichtlich Indikation/Patientenselektion, Erfolgsrate und Komplikationen exakte Analysen. Hieraus ergibt sich ein umfassender Überblick über Art und Lokalisation der Aneurysmen sowie über die verwendeten Stentgrafttypen und deren Erfolgs- und Endoleakraten. 
Die Raten bezüglich Erfolg, Teilerfolg und Misserfolg sind aus Gründen der unterschiedlichen Definition mit der Literatur nur begrenzt vergleichbar.

Die Eingriffsletalität liegt vergleichsweise niedrig.

Hinsichtlich der Datenerfassung müssen Verbesserungen in Form von mehr Pflichtangaben und Plausibilitätsprüfungen durchgeführt werden.

\section{Institute}

${ }^{1}$ Institut für Diagnostische und Interventionelle Radologie, Neuroradiologie und Nuklearmedizin, Universitätsklinikum Knappschaftskrankenhaus GmbH, Ruhr-Universität-Bochum

2 Geschäftsstelle Hamburg, BQS-Institut für Patientensicherheit, Hamburg

3 Institut für Diagnostische und Interventionelle Radiologie, Neuroradiologie und Nuklearmedizin, Klinikum München - Harlaching, München

${ }^{4}$ Institut für Klinische Radiologie, Universitätsklinikum Münster

${ }^{5}$ Radiologie, Asklepios Klinik Hamburg

${ }^{6}$ Klinik für Diagnostische und Interventionelle Radiologie, Universitätsklinikum des Saarlands, Homburg/Saar

\section{Literatur}

1 Heuser L, Arnold CN, Morhard D et al. DeGIR Qualitätsreport 2011 - Bericht über die Behandlungsqualität minimalinvasiver Methoden. Teil 1: Verfahren zur Rekanalisation eingeengter und verschlossener Arterien. Fortschr Röntgenstr 2012; 184: 570-576

2 Bücker A, Gross-Fengels W, Haage P et al. Qualifizierungsleitlinie der Deutschen Röntgengesellschaft (DRG) und der Deutschen Gesellschaft für Interventionelle Radiologie und minimalinvasive Therapie (DeGIR) zur Durchführung interventionell-radiologischer minimalinvasiver Verfahren an Arterien und Venen. Fortschr Röntgenstr 2012; 184: $565-569$

3 Helmberger T. Interventionelle Radiologie - eine Standortbestimmung. Fortschr Röntgenstr 2011; 183: 796 - 798

4 Society of Interventional Radiology Standards of Practice Committee. Guidelines for Percutaneous Transluminal Angioplasty. J Vasc Interv Radiol 2003; 14: S209

5 Santosa F, Moysidis Th, Nowak The et al. Endovascular abdominal aneurysm repair: trends in Germany. Vasa 2012; 41: 268-274

6 Ketelsen D, Bail D, Heuschmid M et al. A Technical Approach for Transfemoral Managing of Hostile Angulated Pelvic Vessel Access in Abdominal Endovascular Aneurysm Repair: A New Technique for Establishing a Buddy Wire. Fortschr Röntgenstr 2012; 184: 899-904

7 van Keulen JW, de Vries JPM, Dekker $H$ et al. One-year multicenter results of 100 abdominal aortic aneurysm patients treated with the Endurant stent graft. J Vasc Surg 2011; 54: 609-615

8 Pitton MB, Schweitzer H, Herber S et al. Endovaskuläre Therapie von abdominellen Aortenaneurysmen: Klinisch-radiologische Ergebnisse im mittelfristigen Verlauf. Fortschr Röntgenstr 2003; 175: 1392

9 Kvinlaug KE, Lawlor DK, Forbes TL et al. Early results from a Canadian multicenter prospective registry of the Endurant stent graft for endo- vascular treatment of abdominal aortic aneurysms. J Endovasc Ther 2012; 19: 58-66

10 Stokmans RA, Teijink JA, Forbes TL et al. Early Results from the ENGAGE Registry: Real-world Performance of the Endurant Stent Graft for Endovascular AAA Repair in 1262 Patients. Eur J Vasc Endovasc Surg 2012; 44: 369-375

11 Freyrie A, Gargiulo M, Fargion A et al. Anaconda(TM) Italian Registry Participating Physicians. AnacondaTM: the Italian Registry. Study protocol and preliminary perioperative results. J Cardiovasc Surg 2011; 52: $629-635$

12 Thomas SM, Beard JD, Ireland $M$ et al. Results from the prospective registry of endovascular treatment of abdominal aortic aneurysms (RETA): mid term results to five years. Eur J Vasc Endovasc Surg 2005; 29: 563-570

13 Leurs LJ, Kievit J, Dagnelie PC et al. EUROSTAR Collaborators. Influence of infrarenal neck length on outcome of endovascular abdominal aortic aneurysm repair. J Endovasc Ther 2006; 13: 640-648

14 Hahne JD, Peitsmeyer P, Habermann C. Erfolgreiche konservative Therapie eines infizierten, perkutan implantierten, infrarenalen Aortenstents. Fortschr Röntgenstr 2011; 183: 173-175

15 Rayt HS, Sutton AJ, London NJM et al. A systemic review and meta-analysis of Endovascular Repair (EVAR for ruptured abdominal aortic aneurysm). Eur J Vasc Endovasc Surg 2008; 36: 536- 544

16 Chambers $D$, Epstein $D$, Walker $S$ et al. Endovascular stents for abdominal aortic aneurysms:a systematic review and economic model. Health Technol Assess 2009; 13: 1

17 Edwards MS, Andrews JS, Edwards AF et al. Results of endovascular aortic aneurysm repair with general, regional, and local/monitored anesthesiacare in the American College of Surgeons National Surgical Quality Improvement Program database. J Vasc Surg 2011; 29: 1097

18 Giles KA, Pomposelli F, Hamdan A et al. Decrease in total aneurysmrelated deaths in the era of endovascular aneurysm repair. J Vasc Surg 2009; 49: 543

19 Lovegrove RE, Javid M, Magee TR et al. A meta-analysis of 21,178 patients undergoing open orendovascular repair of abdominalaortic aneurysm. Br J Surg 2008; 95: 677

20 Nolz $R$, Teufelsbauer $H$, Asenbaum $U$ et al. Type II endoleaks after endovascular repair of abdominal aortic aneurysms: fate of the aneurysm sac and neck changes during long-term follow-up. J Endovasc Ther 2012; 19: 193-199

21 Thomas SM, Gaines PA, Beard JD. Short-term (30-day) Outcome of Endovascular Treatment of Abdominal Aortic Aneurysm: Results from the Prospective Registry of Endovascular Treatment of Abdominal Aortic Aneurysms (RETA). Eur J Vasc Endovasc Surg 2001; 21: 57-64

22 Lange C, Leurs LJ, Buth J. EUROSTAR collaborators.. et al. Endovascular repair of abdominal aortic aneurysm in octogenarians: an analysis based on EUROSTAR data. J Vasc Surg 2005; 42: 624-630

23 Leurs $L J$, Hobo R, Buth J et al. The multicenter experience with a thirdgeneration endovascular device for abdominal aortic aneurysm repair. A report from the EUROSTAR database. J Cardiovasc Surg 2004; 45: $293-300$

24 Greiner A, Grommes J, Jacobs MJ. The place of endovascular treatment in abdominal aortic aneurysm. Dtsch Arztebl Int 2013; 110: 119-125 